\title{
Resultados preliminares sobre un proceso Sol-Gel para la síntesis de disoluciones precursoras de láminas delgadas ferroeléctricas con estructura de Perovskita laminar
}

\author{
M.L.CALZADA, A.GONZÁLEZ, R.JIMÉNEZ Y J.MENDIOLA \\ Inst. Ciencia de materiales de Madrid (C.S.I.C.). Cantoblanco. 28049 - Madrid
}

\begin{abstract}
Se han preparado por sol-gel soluciones precursoras de $\mathrm{SrBi}_{2} \mathrm{Ta}_{2} \mathrm{O}_{9}$, utilizando como reactivos el etóxido de tántalo y los 2-etilhexanoatos de estroncio y de bismuto. Como disolvente mayoritario se empleó el 1,3-propanodiol. Las soluciones resultantes presentaron una baja velocidad de hidrólisis y condensación, haciendo posible su almacenaje y manipulación en atmósferas no exentas de humedad. Las láminas derivadas de estas soluciones y cristalizadas a $750^{\circ} \mathrm{C}$ presentaron como fase mayoritaria la perovskita laminar de $\mathrm{SrBi}_{2} \mathrm{Ta}_{2} \mathrm{O}_{9}$. La polarización eléctrica de estas láminas puso de manifiesto una moderada actividad ferroeléctrica, con valores de polarización remanente de $\operatorname{Pr} \sim 1 \mu \mathrm{C} / \mathrm{cm}^{2}$.
\end{abstract}

Palabras clave: lámina delgada, sol-gel, perovskita laminar, ferroeléctrico.

Preliminary results about a Sol-Gel process for the synthesis of precursor solutions of ferroelectric layered perovskite thin films

$\mathrm{SrBi}_{2} \mathrm{Ta}_{2} \mathrm{O}_{9}$ precursor solutions have been prepared by sol-gel using, as reactives, tantalum ethoxide and strontium and bismuth 2-ethyl-hexanoates. These compounds were disolved in 1,3-propanediol as majoritary solvent. The resulting solutions had low hydrolysis and condensation, thus making possible their manipulation and storage in atmospheres with moisture. Films deposited from these solutions were crystallised at $750^{\circ} \mathrm{C}$, developing, as majoritary phase, the $\mathrm{SrBi}_{2} \mathrm{Ta}_{2} \mathrm{O}_{9}$ layered perovskite. Electric poling of these films indicates a moderate ferroelectric response with values of remanent polarisation of $\operatorname{Pr} \sim 1 \mu \mathrm{C} / \mathrm{cm}^{2}$.

Keyworkds: thin film, sol-gel, layered perovskite, ferroelectric.

\section{INTRODUCCION}

Las láminas delgadas ferroeléctricas con estructura tipo Aurivillius [capas de óxido de bismuto entre las que se encuentran intercaladas capas de perovskita], depositadas sobre substratos de silicio con electrodos de platino, han suscitado un gran interés durante los últimos años debido a su actividad ferroeléctrica y a su baja fatiga (1).

Las perovskitas laminares presentan como fórmula general $\mathrm{A}_{\mathrm{n}-1} \mathrm{Bi}_{2} \mathrm{O}_{3 \mathrm{n}+3^{\prime}}$ dónde, en el caso de su aplicación en memorias no volátiles (NVFERAM), la composición más utilizada es aquella en que $\mathrm{A}=\mathrm{Sr}, \mathrm{B}=\mathrm{Ta}$ y $\mathrm{n}=2$. Esta composición (SBT) tiene una alta temperatura de cristalización que dificulta su integración con la tecnología del silicio (2).

Entre los distintos métodos de preparación de lámina delgada, se encuentra el depósito por sol-gel. Las disoluciones sintetizadas por sol-gel se caracterizan por su alta homogeneidad y reactividad química, que hacen posible reducir la temperatura de cristalización de las láminas amorfas depositadas a partir de ellas (3). Para que una solución sintetizada por sol-gel se pueda utilizar en el depósito de lámina delgada, ésta no debe contener precipitado y tiene que tener una baja velocidad de gelación. Estos requerimientos son a veces difíciles de conseguir, sobre todo, cuando el sol contiene elementos de transición, como es el caso de la mayoría de las composiciones cerámicas ferroeléctricas (titanatos, zirconatos, niobatos o tantalatos). Las soluciones que contienen alcóxidos de elementos de transición reaccionan rápidamente con el agua, dando lugar a la precipitación de sus hidróxidos. Para evitar ésto, y con ello la pérdida de homogeneidad y estequiometría, las soluciones de compuestos ferroeléctricos, sintetizadas por sol-gel se preparan y almacenan en atmósferas exentas de agua.

El método sol-gel más utilizado para la preparación de láminas delgadas ferroeléctricas de titanatos y/o zirconatos de plomo, usa el metoxietanol como disolvente de los alcóxidos (4). Para preservar la homogeneidad de la solución sintetizada con este sistema químico, hay que trabajar en atmósfera seca. Además, el metoxietanol es un compuesto muy tóxico.

La utilización del metoxietanol como disolvente se ha trasvasado a las composiciones ferroeléctricas, preparadas en forma de lámina delgada, con estructura Aurivillius. Este alcohol junto con hidrocarburos aromáticos y ésteres son los disolventes empleados en la literatura [5], en los que los alcóxidos de tántalo o de niobio se disuelven en atmósfera seca.

La forma de evitar la utilización de atmósfera seca durante la síntesis de estas soluciones es buscar ligandos que sean capaces de sustituir grupos alcóxido unidos al metal y que, por su tamaño, lo apantallen impidiendo el ataque de compuestos como el agua. Así, la formación de posibles heterogeneidades en la disolución disminuye, al reducirse su velocidad de hidrólisis y condensación.

En este trabajo se presentan los primeros resultados obtenidos en láminas de SBT obtenidas a partir de un método sol-gel que permite procesar las soluciones precursoras en atmósferas no secas e incrementar la homogeneidad y reactividad del sistema químico. 


\section{EXPERIMENTAL}

\subsection{Preparación de láminas delgadas}

Se ha sintetizado una solución precursora con la composición $\mathrm{SrBi}_{2} \mathrm{Ta}_{2} \mathrm{O}_{9}(\mathrm{SBT})$, utilizando el esquema de la Figura 1. Para ello se sintetizan en una primera etapa las soluciones precursoras de los correspondientes óxidos $\left(\mathrm{SrO}, \mathrm{Bi}_{2} \mathrm{O}_{3}\right.$ y Ta $\mathrm{Ta}_{5}$ ).

La solución de $\mathrm{Sr}$ (II) se obtuvo mediante la disolución de 2etil-hexanoato de estroncio, $\mathrm{Sr}\left(\mathrm{C}_{7} \mathrm{H}_{15} \mathrm{COO}\right)_{2}$, en 1,3-propanodiol, $\mathrm{C}_{3} \mathrm{H}_{6}(\mathrm{OH})_{2}$, utilizando una relación molar del diol al compuesto de $\mathrm{Sr}(\mathrm{II})$ de $\mathrm{R} \sim 15$. A esta mezcla se le incorporó ácido 2-etil-hexanoíco, $\mathrm{C}_{7} \mathrm{H}_{5} \mathrm{COOH}$, en relación molar del ácido al compuesto de $\mathrm{Sr}$ (II) de R 5. Esta mezcla se mantuvo a reflujo en aire durante 8 horas y a continuación se realizó una destilación a la presión de $\sim 0.3 \mathrm{~atm}$, obteniendo una gelatina soluble en etanol, $\mathrm{C}_{2} \mathrm{H}_{5} \mathrm{OH}$. La solución obtenida tuvo una concentración de $\sim 0.3 \mathrm{~mol} / 1$ y una densidad de $\sim 1.2 \mathrm{~g} / \mathrm{cm}^{3}$ y era estable en aire.

La solución de $\mathrm{Bi}(\mathrm{III})$ se preparó disolviendo 2-etil-hexanoato de bismuto, $\mathrm{Bi}\left(\mathrm{C}_{7} \mathrm{H}_{15} \mathrm{COO}\right)_{3^{\prime}}$ en ácido 2-etil-hexanoíco, utilizando una relación molar del ácido al compuesto de Bi(III) de $\mathrm{R} \sim 15$. La mezcla se mantuvo a reflujo durante 3 horas y a continuación se realizó una destilación a la presión de $\sim 0.3 \mathrm{~atm}$. La solución obtenida tuvo una concentración de $\sim 0.3$ moles $/ 1$ y una densidad de $\sim 1.1 \mathrm{~g} / \mathrm{cm}^{3}$. Esta solución se almacenó en aire.

La solución de $\mathrm{Ta}(\mathrm{V})$ se preparó mezclando en atmósfera de nitrógeno, etóxido de tántalo, $\mathrm{Ta}\left(\mathrm{OC}_{2} \mathrm{H}_{5}\right)_{5}$, y 1,3-propanodiol, $\mathrm{C}_{3} \mathrm{H}_{6}(\mathrm{OH})_{2}$, en relación molar del diol al compuesto de $\mathrm{Ta}(\mathrm{V})$ de $\mathrm{R}$ 15. A continuación esta mezcla se sacó al aire y se puso a reflujo durante $8 \mathrm{~h}$, realizando al cabo de este tiempo una destilación a presión atmosférica. La solución obtenida tuvo una concentración de $\sim 0.6$ moles $/ 1$ y una densidad de $\sim 1.4 \mathrm{~g} / \mathrm{cm}^{3}$. Esta solución se mantuvo estable en aire durante meses.

Se tomaron alíquotas de estas soluciones para la obtención de una composición nominal de $\mathrm{SrBi}_{2} \mathrm{Ta}_{2} \mathrm{O}_{9}$. Estas se mezclaron y se calentaron a $\sim 55^{\circ} \mathrm{C}$ a la presión de $\sim 0.3 \mathrm{~atm}$. A esta temperatura empezaron a destilar los productos secundarios de reacción. Cuando la temperatura comenzó a bajar se quitó el calentamiento, obteniéndose una solución precursora de SBT estable en aire, que se diluyó en 2-etil-hexanol.

La solución diluída se depositó sobre substratos de $\mathrm{Pt} / \mathrm{TiO}_{2} / \mathrm{SiO}_{2} / \mathrm{Si}(100)$, mediante la técnica de "spin-coating", con una velocidad de giro de $2000 \mathrm{rpm}$ durante $45 \mathrm{~s}$. El depósito se secó a $\sim 350^{\circ} \mathrm{C}$ durante $60 \mathrm{~s}$. Este proceso se repitió cinco

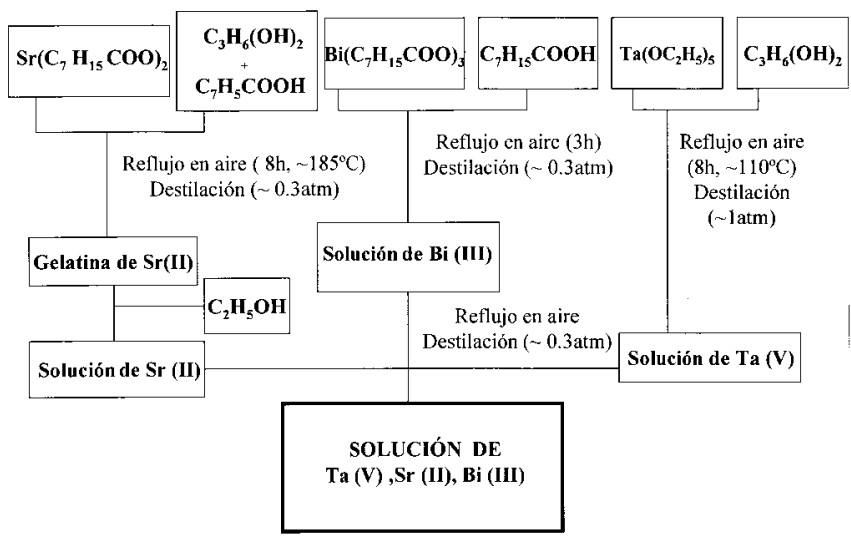

Figura 1. Esquema de síntesis de las soluciones de $\mathrm{SrBi}_{2} \mathrm{Ta}_{2} \mathrm{O}_{9}$.

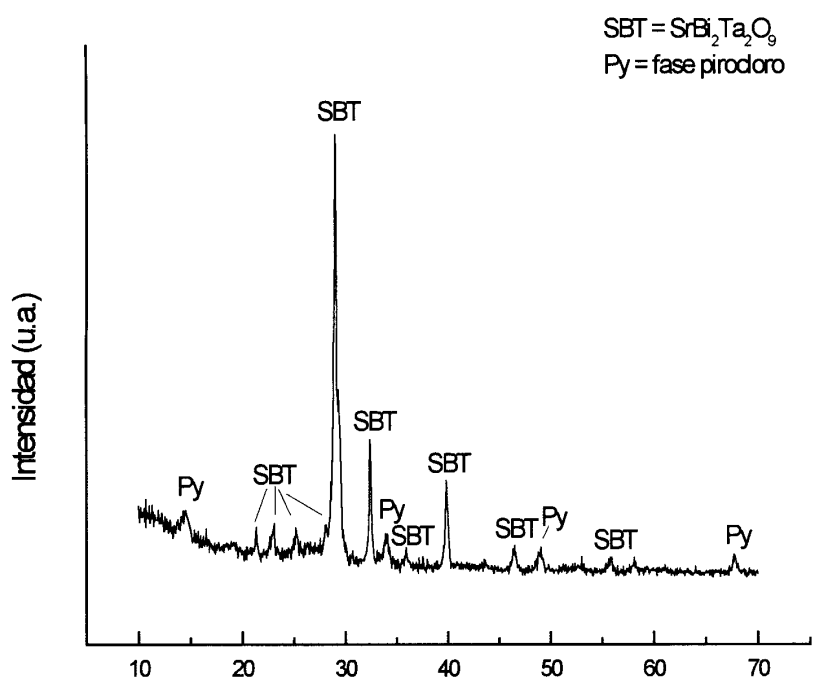

Figura 2. Patrón de difracción de una lámina de $\mathrm{SrBi}_{2} \mathrm{Ta}_{2} \mathrm{O}_{9}$, obtenido mediante difracción de rayos $\mathrm{X}$ con ángulo rasante, utilizando un ángulo de incidencia de $\alpha=2^{\circ}$. BST $=$ perovskita laminar de $\mathrm{SrBi}_{2} \mathrm{Ta}_{2} \mathrm{O}_{9}$ y $\mathrm{Py}=$ fase pirocloro.
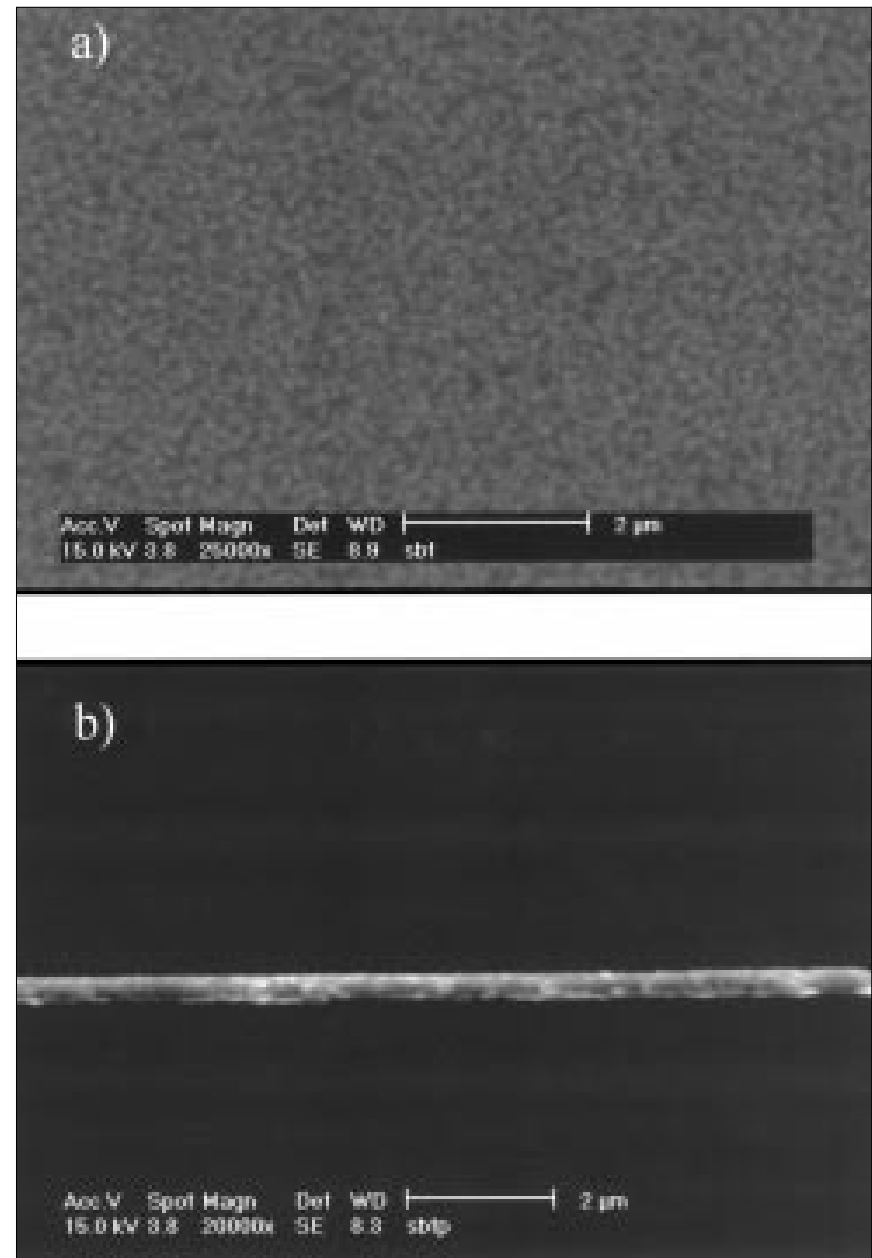

Figura 3. a) Imagen de la superficie de una lámina delgada de $\mathrm{SrBi}_{2} \mathrm{Ta}_{2} \mathrm{O}_{9}$ cristalizada a $750^{\circ} \mathrm{C}$. b) Imagen de una sección transversal de la lámina anterior. Estas láminas se están llevando a cabo en la actualidad. 
veces. La cristalización de la película seca se llevó a cabo en atmósfera enriquecida en oxígeno a una temperatura de $\sim 750^{\circ} \mathrm{C}$ durante $600 \mathrm{~s}$, utilizando una velocidad de calentamiento de $\sim 8^{\circ} \mathrm{C} / \mathrm{s}$. El espesor de la lámina cristalina medido por perfilometría resultó ser de $\sim 400 \mathrm{~nm}$.

La cristalinidad de las láminas de SBT se estudió mediante difracción de rayos X con ángulo rasante (GIXRD, del inglés grazing incidence $X$-ray difraction), utilizando un ángulo de incidencia de $\alpha=2^{\circ}$.

La microscopía electrónica de barrido (MEB) se utilizó para la observación de la microestructura de las láminas cristalinas.

\subsection{Caracterización ferroeléctrica}

Se han medido ciclos de histéresis empleando el equipo comercial de Radiant Technology mode RTA 66A, que usa ondas triangulares de $20 \mathrm{~V}$ de amplitud y $200 \mathrm{~Hz}$ de frecuencia. Con el mismo equipo y empleando el método de los cinco pulsos triangulares, se ha medido la carga conmutada, utilizando una anchura de pulso de 2 y 190 ms, y amplitudes variables. De este modo se puede calcular la polarización conmutada remanente, $\delta \mathrm{P}_{\mathrm{r}}=\mathrm{P}_{\mathrm{r}}^{*}-\mathrm{P}_{\mathrm{r}}^{\wedge}$ y $\delta \mathrm{P}=\mathrm{P}^{*}-\mathrm{P}^{\wedge}$ (ver significado de estos parámetros en Ref.6).

\section{RESULTADOS Y DISCUSIÓN}

Mehrota y col. $(7,8)$ pusieron de manifiesto durante las décadas de los 60 y los 70 la capacidad que tenían determinados alcóxidos metálicos para reaccionar e intercambiar ligandos con alcoholes dihidroxílicos (dioles o glicoles). En este sentido, los pentóxidos de $\mathrm{Nb}$ y de Ta intercambian sus grupos etóxidos con glicoles, dando lugar a derivados glicolados cíclicos con aspecto de líquido viscoso o semi-sólido (sol o gel) [8]. Estos derivados son resistentes al ataque de agentes nucleofílicos, debido al efecto estérico que los ligandos glicolados tienen sobre el catión metálico. Esta característica del etóxido de Ta es la que hace posible en este trabajo, sintetizar un sol de Ta estable en aire, que después de reaccionar con las soluciones de Bi y de Sr, conduce a soluciones precursoras de SBT, en las que se forma un polímero inorgánico que contiene los tres

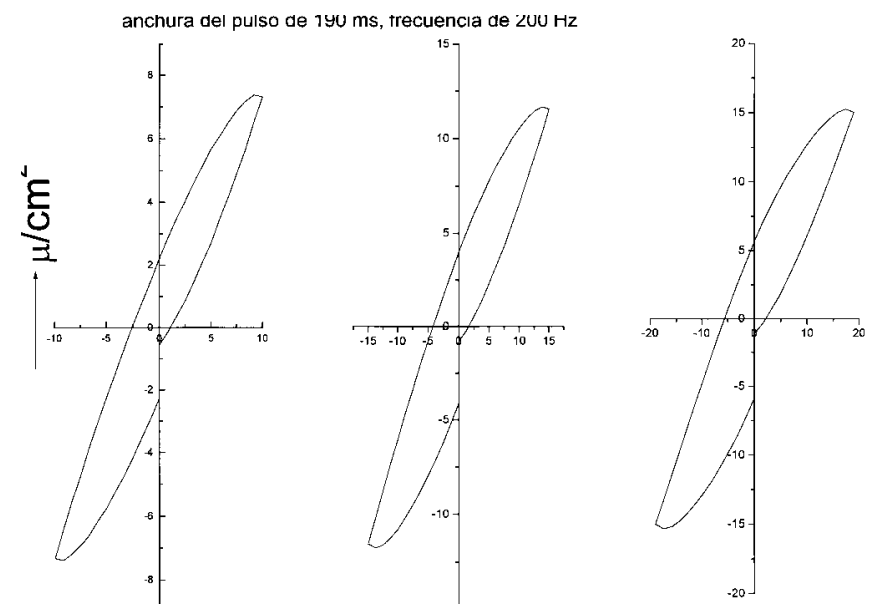

Figura 4: ciclos de histéresis obtenidos aplicando para polarizar campos eléctricos de hasta $20 \mathrm{~V}$. Anchura del pulso de $190 \mathrm{~ms}$, frecuencia de $200 \mathrm{~Hz}$. cationes, y que tiene una baja velocidad de hidrólisis y condensación, por lo que estas soluciones son más homogéneas, y por tanto más reactivas, que soluciones análogas preparadas por otras vías sintéticas.

El aumento de reactividad de estas soluciones se pone de manifiesto en que las láminas depositadas a partir de ellas desarrollan, como fase mayoritaria, la perovskita laminar de SBT a temperaturas $\sim 750^{\circ} \mathrm{C}$ (Figura 2). La fase secundaria, Py, que se observa en el difractograma probablemente se debe a la utilización de la composición estequiométrica en las soluciones precursoras. Debido a la volatilidad del Bi durante el tratamiento térmico de las láminas, se prevé la necesidad de ajustar las relaciones catiónicas, enriqueciendo las soluciones en Bi, para evitar así la formación de fases secundarias (9). Esto redundará en unas mejores prestaciones ferroeléctricas.

La Figura 3 muestra las imágenes de la superficie de la lámina cristalina (Figura 3a) y de su sección transversal (Figura 3b). Esta última micrografía muestra la baja rugosidad de la película. La poca nitidez de la imagen de su superficie sólo permite indicar que estas láminas presentan un pequeño tamaño de grano, < $100 \mathrm{~nm}$. Este pequeño tamaño de grano probablemente sea parcialmente responsable del comportamiento ferroeléctrico de estos materiales.

En la Figura 4 se representan los ciclos de histéresis obtenidos aplicando voltajes de hasta $20 \mathrm{~V}$. A pesar de comprobarse
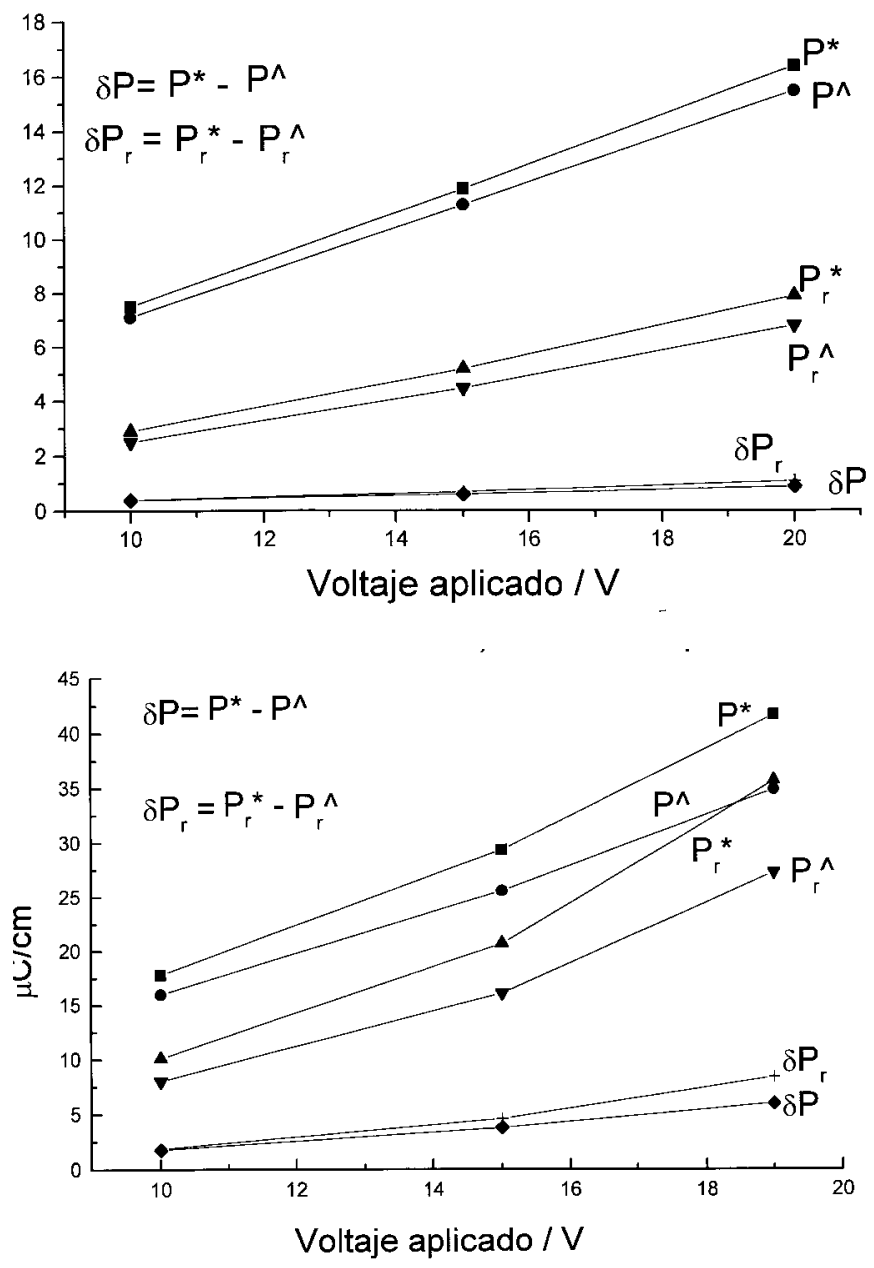

Figura 5: Carga conmutada medida con el método de los cinco pulsos utilizando a) una anchura de pulso de $2 \mathrm{~ms}$ y b) una anchura de pulso de $190 \mathrm{~ms}$. 
que estos ciclos no están saturados, probablemente debido a que el campo efectivo no corresponde al aplicado por la existencia de segundas fases o intercaras, se puede apreciar un valor de polarización remanente de $\sim 5 \mu \mathrm{C}^{\mathrm{cm}} \mathrm{cm}^{-2}$, resultado de una fuerte despolarización.

En la Figura 5 se muestran los resultados obtenidos con el método de pulsos. Se comprueba que los valores de $\mathrm{dP}$ y $\mathrm{dP}_{\mathrm{r}}$ son muy dependientes, no sólo del campo aplicado, sino de la anchura del pulso, de modo que cuanto mayor es éste, a igualdad de amplitud, la carga conmutada es también mayor: para una anchura de $190 \mathrm{~ms}, \delta \mathrm{P}_{\mathrm{r}} \sim 8.4 \mu \mathrm{C} . \mathrm{cm}^{-2}$, mientras que $\delta \mathrm{P}_{\mathrm{r}} \sim 1.1$ $\mu \mathrm{C} . \mathrm{cm}^{-2}$ para $2 \mathrm{~ms}$ de anchura. Este comportamiento puede significar que la conmutación es lenta o bien, que existen diferentes tiempos de conmutación, propio de materiales en los que el tamaño de grano es menor que el espesor de la lámina, como es el presente caso.

A pesar de que estas láminas no presentan las mejores prestaciones ferroeléctricas, si que ponen de manifiesto el grado de aproximación al material deseado con el nuevo procesado propuesto.

\section{CONCLUSIONES}

Se ha desarrollado un proceso sol-gel para la síntesis de soluciones precursoras de composiciones ferroeléctricas con estructura de perovskita laminar. Este proceso aprovecha la reactividad de los alcóxidos de los elementos del grupo $\mathrm{Vb}$ con los glicoles, para formar soluciones estables en aire y con alta reactividad. Las láminas delgadas de SBT preparadas a partir de estas soluciones presentan una moderada actividad ferroeléctrica.

\section{AGRADECIMIENTOS}

Este trabajo ha sido financiado por la CICYT a través del proyecto MAT98-1068. Agradecemos al Dr. Alejandro Várez, la realización de las micrografías que se presentan en este trabajo.

\section{BIBLIOGRAFÍA}

1. Auciello, O.; Scott, J.F. and Ramesh, R., "The physics of ferroelectric memories". Physics Today, 7 22-27 (1998).

2. Kato, K.; Finder, J.M.; Dey, S.K. and Torii Y., "Low-temperature crystallization and ferroelectric properties of sol-gel derived layer-structured perovskite thin films". Integrated Ferroelectrics, 18 237-247 (1997).

3. Schwartz, R.W.,"Chemical solution deposition of perovskite thin films".Chem.Mater., 9 2325-2340 (1997).

4. Budd, K.D.; Dey, S.K. and Payne, D.A., "The effect of the hydrolysis conditions on the characteristics of $\mathrm{PbTiO}_{3}$ gels and thin films". Mater.Res.Soc.Symp.Proc., 73 711-716 (1986).

5. Paz de Araujo, C.A.; Cuchiaro, J.D.; Scott, M.C. and McMillan, L.D., "Process for fabricating layered superlattice materials". International Application Patent, $\mathrm{n}^{\circ}$ WO 93/12538. USA, June 1993.

6. Mendiola, J.; Ramos, P and Calzada, M.L. "Effect of pretreatments on the ferroelectricity of $(\mathrm{Pb}, \mathrm{Ca}) \mathrm{TiO}_{3}$ thin films". J.Phys.Chem.Solids, 59(9) 1571-78 (1998).

7. Mehrotra, R.C. and Kapoor, P.N.; "Organic compounds of niobium. V. Reaction of niobium pentaethoxide with glycols". J.Less-Common Metals, 8 419-427 (1965).

8. Bradley, D.C.; Mehrotra, R.C. and Gaur, D.P., "Metal alkoxides”. Ed. by Academic Press Inc.Ltd., London (1978), pp.192-194.

9. Chen T.C.; Li T.; Zhang, X. And Desu B., "The effect of excess bismuth on the ferroelectric properties of $\mathrm{SrBi}_{2} \mathrm{Ta}_{2} \mathrm{O}_{9}$ thin films". J.Mater.Res., 12(6) 1569-75 (1997). 